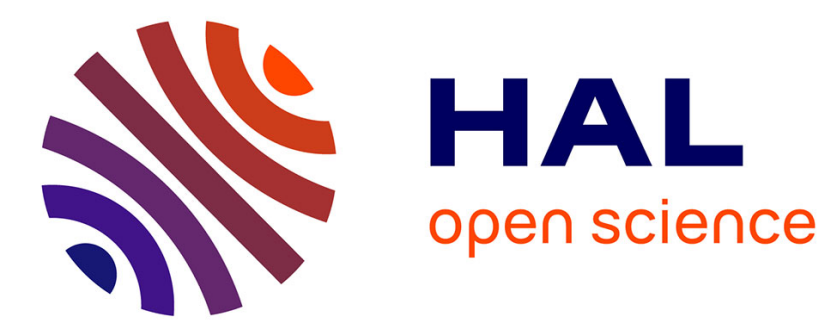

\title{
Spectroscopic studies of GTA welding plasmas. Temperature calculation and dilution measurement
}

\author{
David Lacroix, C Boudot, G Jeandel
}

\section{To cite this version:}

David Lacroix, C Boudot, G Jeandel. Spectroscopic studies of GTA welding plasmas. Temperature calculation and dilution measurement. European Physical Journal: Applied Physics, 1999, 8 (1), pp.61 - 69. 10.1051/epjap:1999230 . hal-01887303

\section{HAL Id: hal-01887303 \\ https://hal.science/hal-01887303}

Submitted on 3 Oct 2018

HAL is a multi-disciplinary open access archive for the deposit and dissemination of scientific research documents, whether they are published or not. The documents may come from teaching and research institutions in France or abroad, or from public or private research centers.
L'archive ouverte pluridisciplinaire HAL, est destinée au dépôt et à la diffusion de documents scientifiques de niveau recherche, publiés ou non, émanant des établissements d'enseignement et de recherche français ou étrangers, des laboratoires publics ou privés. 
Eur. Phys. J. AP 8, 61-69 (1999)

\title{
Spectroscopic studies of GTA welding plasmas. Temperature calculation and dilution measurement
}

\author{
D. Lacroix $^{1, a}$, C. Boudot ${ }^{2}$, and G. Jeandel ${ }^{1}$ \\ 1 LEMTA, Université Henri Poincaré, B.P. 239, 54506 Vandœuvre-lès-Nancy Cedex, France \\ 2 FRAMATOME, Centre Technique, Z.I.P. Sud, B.P. 13, 71380 St. Marcel Cedex, France
}

Received: 11 March 1999 / Revised: 7 July 1999 / Accepted: 29 July 1999

\begin{abstract}
A spectroscopic study of the GTAW plasma-plume created during the welding of stainless steel and other materials (iron, nickel and chromium) has been carried out. The spectra of these plasmas have been studied for several welding parameters. Temperature calculations are based on the observation of relative intensities and shapes of the emission peaks. We assume that the plasma is in local thermal equilibrium. The temperature is calculated with the Boltzmann plot method from twelve iron emission lines (in the range 368-385 $\mathrm{nm}$ ): it varies between 9650 and $12100 \mathrm{~K}$. Dilution experiments have been carried out. We checked the mixing of metals: during welding of two different metallic plates and during welding with an Inconel wire. Dilution is monitored following the intensity of some characteristic emission lines (chromium and nickel). Comparison of spectroscopic results and metallographic ones is made.
\end{abstract}

PACS. 52.70.-m Plasma diagnostic techniques and instrumentation - 52.75.-d Plasma devices and applications - 83.85.-c Techniques and apparatus

\section{Introduction}

The utilisation of gas-tungsten arc-welding (GTAW) and gas-metal arc-welding (GMAW) devices for steel welding is old and very widespread. These joining techniques are well known and often used. However the physical phenomena occurring during arc-welding are not entirely understood, especially concerning the plasma arc and the weld pool behaviour. Recently, several studies have been carried out on these subjects. Some have dealt with the prediction of the arc-welding process through mathematical models [1-4]. In these papers, heat-flow, fluid-flow and electromagnetic phenomena encountered in GTAW and GMAW are described, solving the transport equations for the arc (conservation of mass, axial momentum, radial momentum, energy and charge continuity) by iterative schemes. The calculated results give temperature and shape of the arc plasma. Furthermore, in the case of GMAW, they explain the droplet formation at the anode area (consumable electrode) and allow estimating the mass flow deposit. These authors $[1,4]$ have computed temperatures in the plasma arc which typically vary between 10000 and $24000 \mathrm{~K}$ according to the processing parameters and the location inside the plume. The current pulse shaping effect on GTAW is also a subject of interest in numerical simulation of welding. Fan et al. [3] showed that the shape of the arc strongly depends on the frequency and on the thermal inertia of the arc. Above $10 \mathrm{kHz}$, the arc is stable as in the case of DC.

\footnotetext{
a e-mail: dlacroix@lemta.sciences.u-nancy.fr
}

Other studies are related to the automation of the welding with descriptions of new sensors and technologies [5-8]. Different techniques can be selected to control arc welding. The most common are based on the light (emitted and reflected) or the sound produced while welding. Lee and $\mathrm{Na}[7]$ have done bidirectional reflectance measurements to estimate the arc light influence on optical sensors used for automated joint tracking. Their experiments and computations showed that arc light reflected at the metallic surface influences considerably the reliability of optical sensors. However, this method can only check the displacement of the welding device, it does not gave any information about the weld seam quality. On-line control of the welds can be achieved from study of the arc welding sound signature as Saini et al. [5] have done. In these experiments of the GMAW process, recording of the sound produced while welding allows monitoring of the arc behaviour, comparing the signal obtained to a reference recording done for an ideal arc behaviour. The frequency analysis of these signals confirms the existence of characteristic peaks which should be related to the presence of defects. However, this hypothesis must be corroborated.

Choice of a suitable shielding gas improving the weld seam quality is also of interest $[9,10]$. Onsøien et al. [9] have studied the influence of hydrogen addition to argon in the shielding gas. Measurements done on A304L stainless steel indicate that the weld depths are significantly influenced (increase in penetration of over 50\%) by increasing the hydrogen content in the shielding gas. 
The aim of this paper is to provide an analysis of the arc-welding process using a spectroscopic device in the ultraviolet and visible ranges in order to find characteristic wavelengths which can be used for welding monitoring. The light emitted by the interaction between the electric arc and the metallic piece can give much information concerning the joining process. Similar investigations have been already done in the case of laser welding where a plasma plume is induced above the workpiece [11].

The first step of the study is to collect data for several welding parameters for different kinds of metals (pure iron, chromium, nickel, stainless steel, ...). Then information about the temperature of the metallic plasma-plume can be found using techniques already employed in the characterisation of laser-welding of steel [12].

The variation of line intensity in the emission spectra of the plasma can also be related to the dilution phenomenon in the case of joining of two different metals, as we show in the second section of this article. In the last part of the paper we present results concerning gas-tungsten arc-welding with metallic wire deposition. This technique is often used in the case of large thickness welding.

\section{Experimental device}

A GTA welding device is used for all experiments. The current intensity is set between 140 and $280 \mathrm{~A}$. The voltage varies in the range of 10 to $12 \mathrm{~V}$. In all experiments the translation speed of the device is 3 inches per minute. A few measurements have been done with a GMA welding machine. Some spectra were recorded, they are much different from those obtained during GTA welding.

The spectrometer is a $460 \mathrm{~mm}$ focal length device (Jobin Yvon HR460) with two gratings, one of 150 lines $/ \mathrm{mm}$, the other of 1800 lines $/ \mathrm{mm}$. The dispersion of the spectrograph is $12 \AA / \mathrm{mm}$ with the 1800 lines $/ \mathrm{mm}$ grating. The detector is a photo-diode array made of 1024 elementary silicon cells $(25 \mu \mathrm{m}$ width and $2.5 \mathrm{~mm}$ height). The entrance slit is fixed at $31.75 \mu \mathrm{m}$. An optical fibre is used to collect the light emitted from the plasma plume. A focusing doublet is set at the extremity of the fibre. This focusing device is linked to the welding machine in order to follow the same part of the plasma-plume when we do the weld. The whole device (spectrograph + detector + entrance slit) is driven by the SPECTRAMAX software.

Different metals were used for the welding experiments. Some are pure materials used to obtain characteristic spectra of the arc plasma; the other metals are those which are usually used in industry. They are (chemical composition):

- iron (99.99\% pure) - plate,

- chromium (99.7\% pure) - plate,

- nickel (99.95\% pure) - plate,

- E24 (steel) - plate,

- 18MND5 (weakly alloyed steel - Mn 1.38\%, Ni 0.61\%, Mo $0.52 \%$ ) - plate,

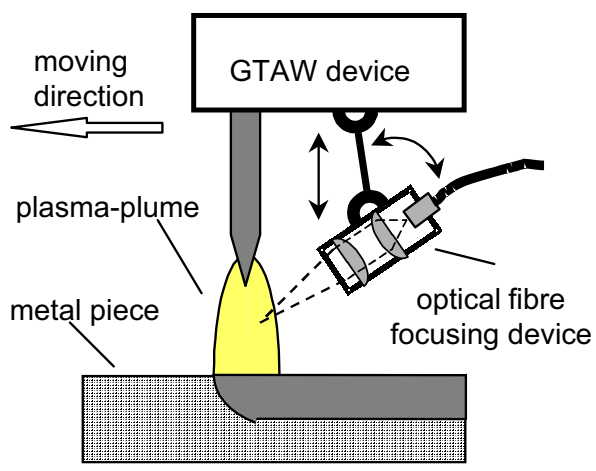

Fig. 1. Experimental device.

- A304L (stainless steel - Fe $\pm 71 \%$, Cr 18.2\%, Ni 8.75\%, Mn $1.49 \%$ ) - plate,

- Inconel 600 (heat resistant alloy - Ni 74.5\%, Cr 15.2\%, Fe $8.3 \%$ ) - plate,

- Inconel 82 (heat resistant alloy - Ni 70.45\%, Cr 20.99\%, Mn 3.04\%, Fe 2.72\%) - wire.

The metallic plates for pure materials are thin $(3 \mathrm{~mm})$. They have been used only to obtain characteristic emission spectra. In order to weld such thin plates we fastened them on large steel pieces. For the other experiments on the steel and the Inconel, the metal plate thickness was between 10 and $15 \mathrm{~mm}$. The shielding gas used in GTAW has also been modified in some experiments. Generally we employed pure argon but in some case we did welds under an argon-hydrogen mixture and under helium. The experimental device is described in Figure 1. We give a schematic description of the weld plate, the arc and the focusing device, which is representative of most cases studied. The typical length of the arc is around five millimetres, but it changes continuously during welding, an electronic device being used to regulate the electrode position for a given current intensity and voltage. The area viewed by the optical fibre is circular and has a diameter of $1.5 \mathrm{~mm}$ in the focal plane, at $31 \mathrm{~mm}$ from the output of the focusing device. This device is made of two plane - concave lenses. It is obvious that we have integrated values of plasma plume light intensity, which give us integrated values of temperature, as we will see further.

\section{Experimental results and discussions}

\subsection{Spectroscopic measurements}

As we indicated in the introduction, the first part of the study was devoted to recording spectra of several pure materials (i.e.: iron, chromium and nickel). This step is necessary due to the great number of emission lines that can be found in the ultraviolet and visible ranges.

For example, we have determined more than 300 iron lines (neutral and singly ionised) between 230 and $550 \mathrm{~nm}$ in the case of GTA welding. Furthermore, we have to consider the lines coming from the shielding gas (argon, nitrogen or helium). This work was done before considering 


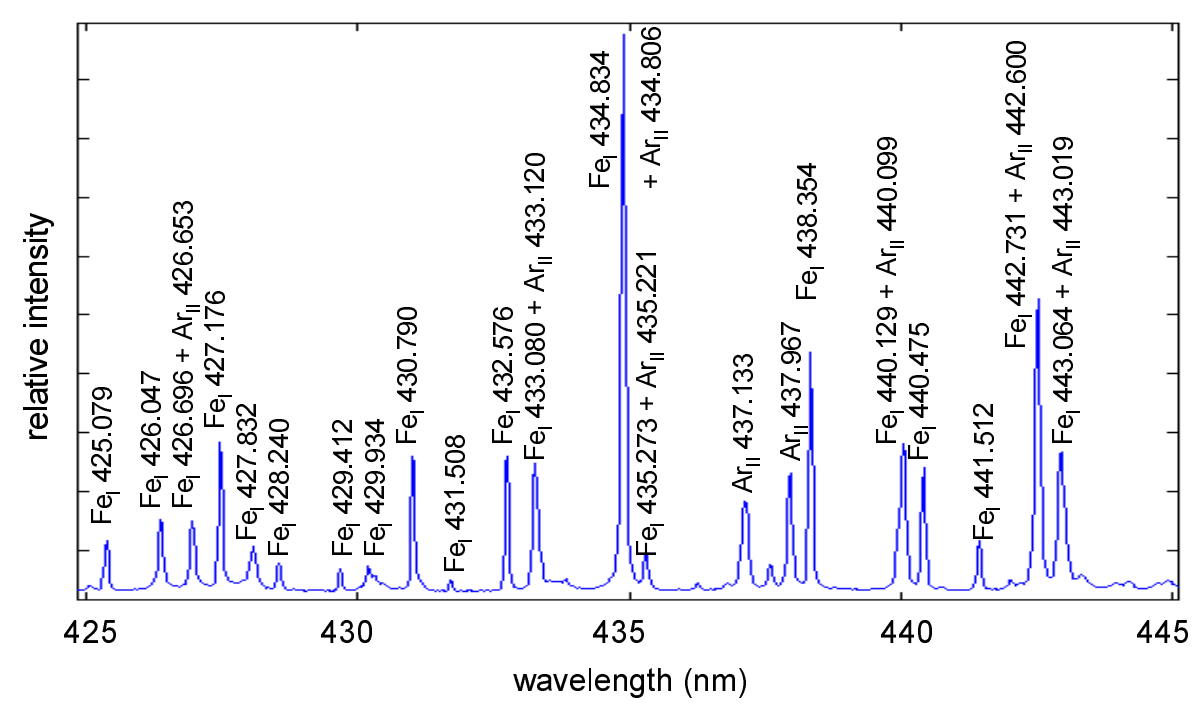

Fig. 2. Stainless steel gas tungsten arc welding spectrum under argon shielding.

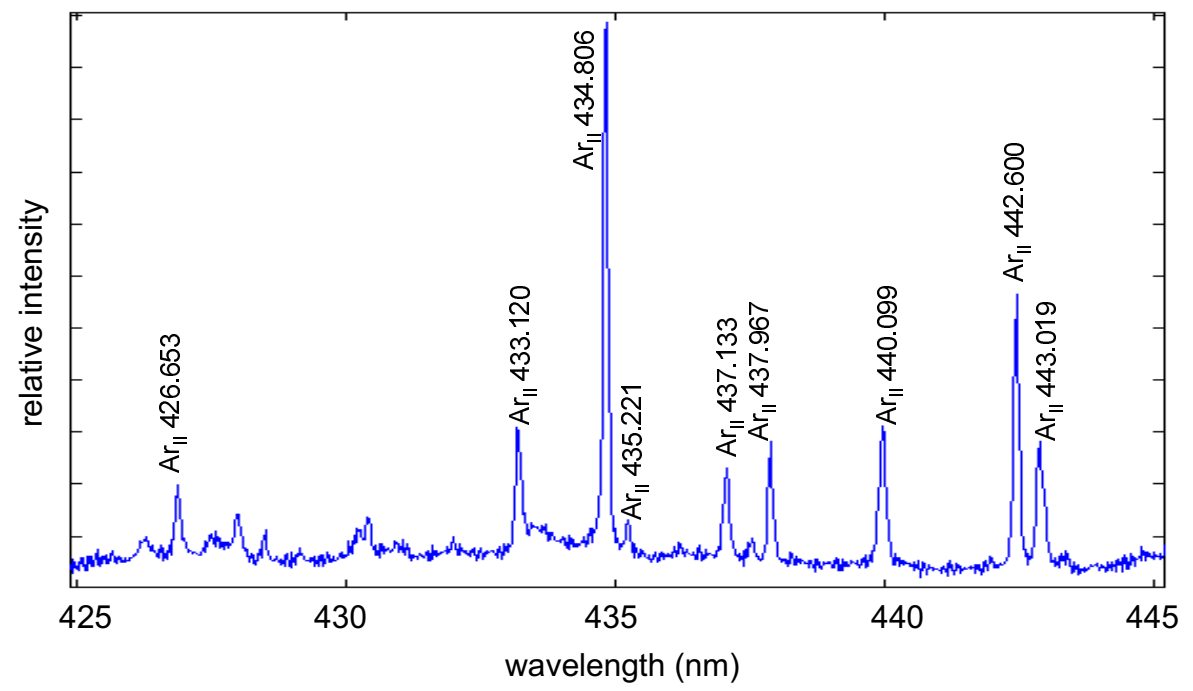

Fig. 3. Argon spectrum for gas tungsten arc welding above a copper plate.

stainless steel and other alloy such as Inconel where other different components exist. In these last cases, the spectra might also contain chromium, nickel, molybdenum and manganese [13]. Figure 2 gives a small portion of the iron spectrum (425-445 nm) obtained during arc welding with argon shielding. The relative intensity of the emission lines is based on the crude data of the PDA detector.

Argon emission lines are more intense than those of iron in GTA welding. This can be confirm by studying several spectra of iron under different shielding gases (helium, argon, argon and helium). The comparison of these spectra clearly point out the predominance of gas emission lines in the spectra, especially in the wavelength range above $400 \mathrm{~nm}$. Furthermore, they are singly ionised lines for wavelengths below $650 \mathrm{~nm}$, the electric arc breaking argon atoms. In the upper part of the plume the metallic species are in small amounts. We observe lines basically due to the shielding gas. In order to characterize these lines, we have made an electric arc between the tungsten electrode (cathode) and a copper target (anode) which is not melted during the process. In this case, there are no metallic vapours, so we only see the gas lines, Figure 3.

We have made recent measurements with a GMA welding device and we present on a same plot the iron spectra under argon shielding for the two different processes (Fig. 4). One can clearly see that the gas-metal arc welding spectrum mainly contain metallic species. The singly ionised argon lines have vanished. Moreover we can on this figure that the GMA spectrum should be hotter due to the more intense metallic emission lines which overlap the iron lines presented in Figure 2.

Other gases such as helium and argon-hydrogen mixtures, have been studied with the same technique. The spectra contain fewer emission lines than in the case of argon. Moreover, the spectral aspects change strongly for similar current parameters. Same remarks have been made 


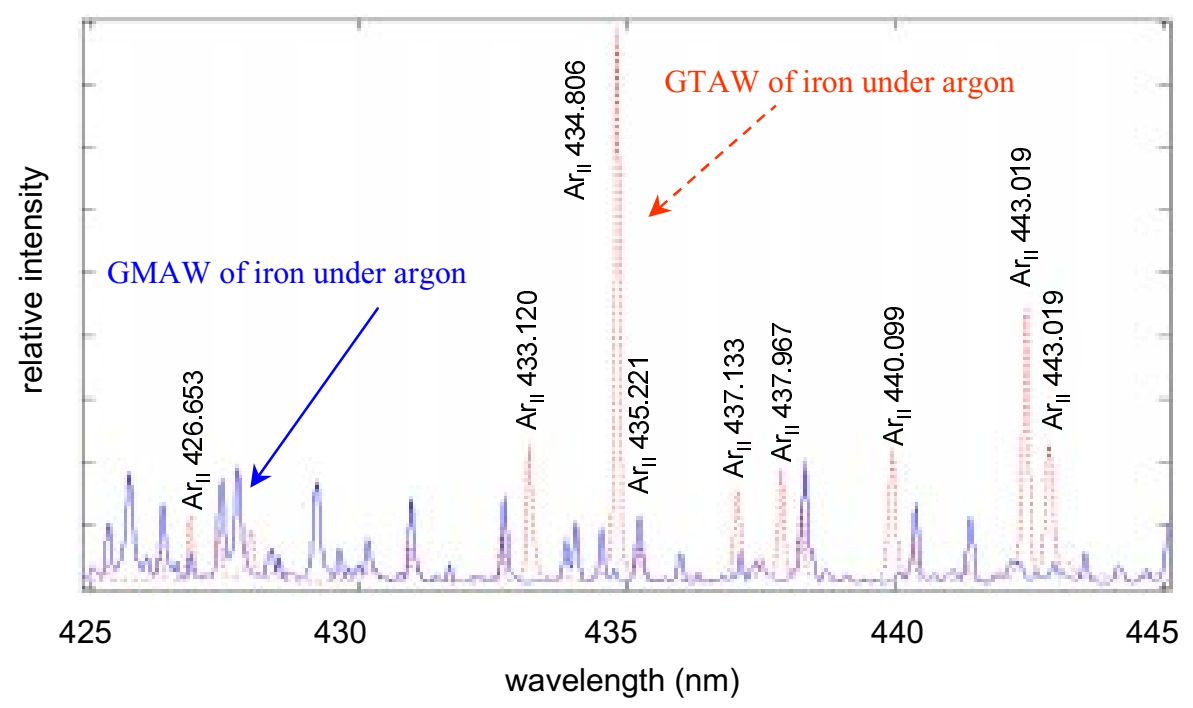

Fig. 4. Iron gas tungsten arc welding (dotted curve) and iron gas metal arc welding (solid curve) spectra under argon shielding.

by Richardson [14] in a paper dealing with spectroscopic measurements of GTAW and GMAW joining processes. In this study, despite the average spectral sensitivity of the acquisition device, the author observed that GTAW spectra are dominated by gas emission lines. The gas plays a primary role in ionising the media. On the other hand, the GMAW spectra are more intense and metallic species seem to be the ionised carriers of the arc.

\subsection{Plasma temperature calculation}

The plasma studied here is assumed to be in Local Thermal Equilibrium (LTE). There is no microscopic reversibility of the collisional and radiative processes in the medium, so Planck's law cannot be applied. The criterion for the LTE is given by Griem [15] and Marr [16]; we must have:

$$
N_{\mathrm{e}} \geq K \sqrt{T_{\mathrm{e}}}\left(E_{m n}\right)^{3}
$$

where $N_{\mathrm{e}}$ is the electron density $\left(\mathrm{cm}^{-3}\right), K$ a collisional coefficient $\left(K \approx 10^{12} \mathrm{eV}^{3} \mathrm{~K}^{-1 / 2} \mathrm{~cm}^{-3}\right)$ which is equal to $K=(5 / 8)\left(\alpha / a_{0} E_{\mathrm{H}}\right)^{3} \sqrt{\pi k / E_{\mathrm{H}}}$ with $\alpha$ the fine structure constant, $a_{0}$ the Bohr radius in $(\mathrm{cm}), k$ the Boltzmann constant in $\left(\mathrm{eV} \mathrm{K}^{-1}\right), E_{\mathrm{H}}$ the ionisation potential of hydrogen in $(\mathrm{eV})$ and $E_{m n}$ the energy transition from the level " $m$ " to " $n$ " $\left(E_{m n}\right.$ is approximately equal to $0.6 \mathrm{eV}$ for iron at $7000 \mathrm{~K}$ ). With these values we find $N_{\mathrm{e}} \geq 1.81 \times 10^{19} \mathrm{~m}^{-3}$, which will be checked in our experiments.

In the case of LTE, we check the Maxwell, the Boltzmann and the Saha laws. The Boltzmann law is used to calculate the temperature of the plasma. The Saha law is employed to determine electron density.

The electron temperature of the plasma plume is established using the relative intensities of several emission lines. On one hand, we have the intensity of an emission line for the transition " $m$ " to " $n$ " $\left(I_{m n}\right)$, which depends on $A_{m n}$ the transition probability in $\left(\mathrm{s}^{-1}\right), N_{m}$ the density at the energy level " $m$ " in $\left(\mathrm{m}^{-3}\right), h$ the Planck's constant in $(\mathrm{J} \mathrm{s})$ and $\nu_{n m}$ the frequency in $\left(\mathrm{s}^{-1}\right)$ :

$$
I_{m n}=N_{m} A_{m n} h \nu_{n m} \text {. }
$$

On the other hand, the Boltzmann law:

$$
\frac{N_{m}}{N}=\frac{g_{m}}{Z(T)} \exp \left(\frac{-E_{m}}{k T}\right)
$$

with $g_{m}$ the statistical weight of the level " $m$ " and $Z(T)$ the partition function. Parameters $A_{m n}, N_{m}, Z(T)$ and $g_{m}$ are tabulated for each emission line.

For a set of chosen emission lines, we can draw the logarithm of $\left(I_{m n} \lambda_{m n} / A_{m n} g_{m}\right)$ versus the energy of the upper state $E_{m}$. The slope of the curve is equal to $-1 / k T$ : it allows electron temperature calculation.

The chosen emission lines must fulfil a criterion on the upper energy levels $E_{m}$ and $E_{p}$ for the wavelengths $\lambda_{m n}$ and $\lambda_{p q}$ which is $E_{m}-E_{p}>k T$.

The emission lines used to do the calculations are taken in the range of $368-385 \mathrm{~nm}$. There are twelve lines which are identified on the A316L spectra. They are iron lines and the upper energy levels of these lines are sufficiently spaced to certify the accuracy of the calculation. On the other hand, the chosen emission lines are close enough to consider a linear correction on the measured intensities due to the PDA detector sensitivity evolution. The spectroscopic parameters of each iron line are given in Table 1 $[17,18]$.

The same computation has been done for the three other metals. We present in Table 2 the temperatures (for similar welding parameters). It can be clearly seen that the pure iron plasma temperature is lower than the others (below $10000 \mathrm{~K}$ ).

In the following part of this section, we present some calculated results. First, we have studied the influence of the nature of the metal on the plasma temperature. We were also interested in the influence of the shielding gas and the arc power supplied. 
Table 1. Fe I line characteristics; $\lambda$ wavelength; $E_{\text {low }}$ lower energy level of the transition; $E_{\mathrm{up}}$ upper energy level of the transition; $g_{i}$ and $g_{k}$ statistical weights; $A_{k i}$ transition probability; $f_{i k}$ oscillator strength.

\begin{tabular}{ccccccc}
\hline$\lambda(\mathrm{nm})$ & $E_{\text {low }}(\mathrm{eV})$ & $E_{\text {up }}(\mathrm{eV})$ & $g_{i}$ & $g_{k}$ & $A_{k i}\left(10^{8} \mathrm{~s}\right)$ & $\log (g f)$ \\
\hline 368.224 & 3.546758 & 6.913111 & 2 & 2 & 1.71 & 0.24 \\
369.401 & 3.038676 & 6.394307 & 2 & 3 & 0.682 & -0.01 \\
370.557 & 0.051572 & 3.396738 & 3 & 3 & 0.0321 & -1.334 \\
372.256 & 0.087291 & 3.417183 & 2 & 2 & 0.0497 & -1.287 \\
373.332 & 0.110121 & 3.430421 & 1 & 1 & 0.0620 & -1.41 \\
374.556 & 0.087291 & 3.396738 & 2 & 3 & 0.115 & -0.771 \\
374.826 & 0.110121 & 3.417183 & 1 & 2 & 0.0915 & -1.016 \\
378.788 & 1.011123 & 4.283597 & 1 & 2 & 0.129 & -0.859 \\
379.500 & 0.990177 & 4.256509 & 2 & 3 & 0.115 & -0.761 \\
381.296 & 0.958221 & 4.209167 & 3 & 2 & 0.0791 & -1.064 \\
385.637 & 0.051572 & 3.265926 & 3 & 2 & 0.0464 & -1.286 \\
385.991 & 0 & 3.211406 & 4 & 4 & 0.0969 & -0.71 \\
\hline
\end{tabular}

Table 2. Plasmas temperatures.

\begin{tabular}{lr}
\hline Metal & Temperature (K) \\
\hline Inconel 600 & 11775 \\
Stainless steel A304L & 11601 \\
Steel E24 & 10617 \\
Pure iron (99.99\%) & 9653 \\
\hline
\end{tabular}

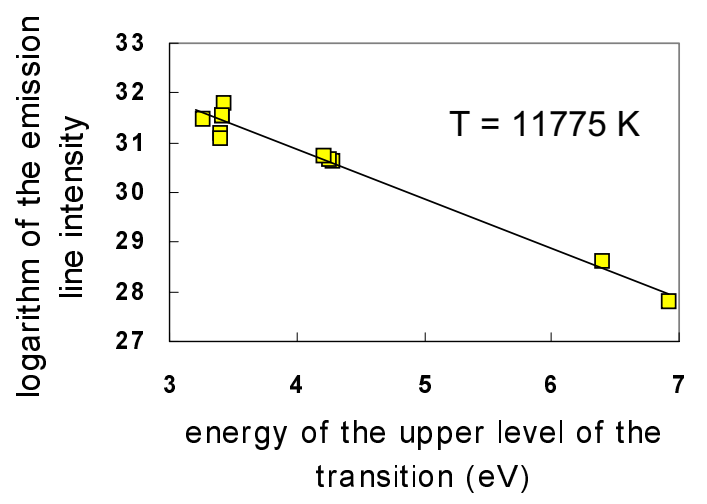

Fig. 5. Boltzmann plot of Inconel plasma; $U=10 \mathrm{~V}, I=$ $210 \mathrm{~A}, v=3 \mathrm{inch} / \mathrm{min}^{-1}$.

\subsubsection{Temperature calculations}

The GTAW processing parameters are: $U=10 \mathrm{~V}, I=$ $210 \mathrm{~A}$ and $v=3 \mathrm{inch} / \mathrm{min}$ ( $v$ translation velocity). The shielding gas used is argon with a flow of $15 \mathrm{l} / \mathrm{min}$.

Figure 5 shows the Boltzmann plot for an Inconel alloy welded with the previous processing parameters $(10 \mathrm{~V}$, 210 A, 3 inch/min). We did not do Abel's inversion on measured emission line intensities. The estimated temperature is equal to $11775 \mathrm{~K}$ with an uncertainty of $\pm 100 \mathrm{~K}$. This uncertainty corresponds to the maximal dif- ference existing between several temperature calculations (four) obtained from welding plasma recorded for identical welding parameters.

In the case of the alloys, the temperature increases, due to the presence of elements such as chromium. The lower ionisation potential of chromium $\left(E_{\infty}^{\mathrm{Cr} 1}=6.77 \mathrm{eV}\right)$ with respect to that of iron $\left(E_{\infty}^{\mathrm{Fe} 1}=7.87 \mathrm{eV}\right)$ renders easier the production of free electrons in the arc induced alloyed plasma, such as Inconel or stainless steel plasmas. The absorption in the plasma plume increases with the free electron density according to the Inverse Bremsstrahlung theory which implies an increase of plume temperature. We have already seen that, in laser welding, where this phenomenon occurs at a lower temperature [12] (around $5000 \mathrm{~K}$ for iron and 6000 to $7000 \mathrm{~K}$ for stainless steel, for a similar supplied power $\approx 2000 \mathrm{~W})$. In the case of GMA welding, the achieved temperatures are higher but it is difficult to compare the two processes. In GMAW the supplied power changes (we set the voltage and the frequency, but the current varies).

We have also modified the output power of the GTAW device. Experiments have been done at higher intensities $250 \mathrm{~A}$ and $280 \mathrm{~A}$, for similar voltages (between 10 and $12 \mathrm{~V})$.

The results show weak variations of the plume temperature. Nevertheless, we observe that the weld seams are larger when we increase the arc power. Contrary to the laser-induced plasma which increases its temperature absorbing the energy of the beam (the absorption of the beam growing with the temperature), the arc welding plasma remains at the same temperature. This observation has already been made in a recent paper by Evans et al. [19] who studied arc plasma parameters in welding.

Other studies on the welding arc temperature show that the plasma plume temperature is very sensitive to the point observed in the plume. With a more accurate focusing device Vervisch et al. [20] have shown that the plume temperature at the centre of the plasma can reach more than $20000 \mathrm{~K}$ and decrease to $5000 \mathrm{~K}$ at the edge of the plume ( $5 \mathrm{~mm}$ from the centre). Our measurements are between these two values, the optical device used to collect the plasma plume light integrating a large part of the plume. However, the purpose of the study is not to determine accurately the plasma temperature but to study the intensity variations which might help us to determine the dilution and defects.

\subsection{Dilution experiments}

The second part of the study is devoted to characterization of dilution problems occurring during the welding of two different metals. Two experiments have been carried out; the first between E24 steel and A304L stainless steel, the second between E24 steel and an Inconel alloy. Spectroscopic measurements have been made in the wavelength range of 367 to $393 \mathrm{~nm}$. The GTAW parameters are $I=210 \mathrm{~A}, U=10 \mathrm{~V}, v=3 \mathrm{inch} / \mathrm{min}$ with argon shielding. Two experimental procedures have been carried out, Figure 6. 


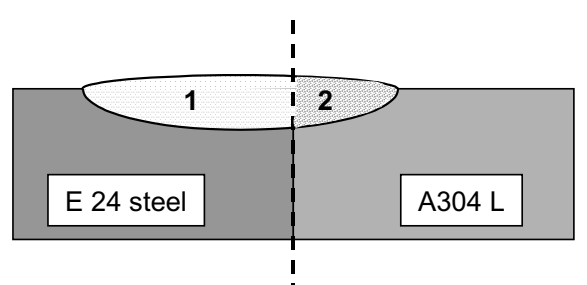

Dilution between 'two metal plates.

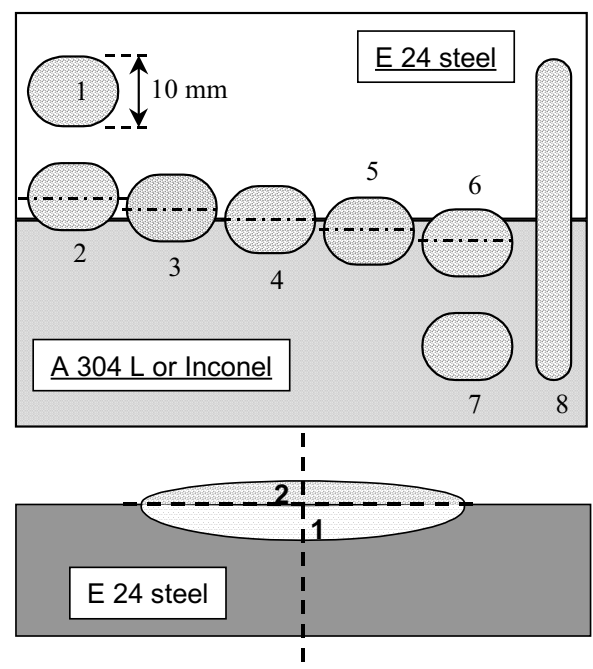

Dilution between a wire and a plate.

Fig. 6. Dilution experiment details.

First, we have done seven welds between the E24 steel and the Inconel or the A304L. The first and the last welds ("1" and "7") contain only one metal. The weld seam "2" and " 6 " axes are at $3 \mathrm{~mm}$ from the edge of the two plates. The weld seam " 3 " and " 5 " axes are at $1.5 \mathrm{~mm}$ from the edge of the two plates. The weld seam " 4 " axis is on the edge of the two plates.

In a second experiment, we record 130 spectra successively, welding the two metal plates according to the seam numbered "8".

We present here results concerning the welding of E24 and A304L stainless steel. Spectroscopic measurements done for each weld seam ("1" to "7") give the plasma temperatures, Figure 7. A metallurgical analysis of the seams has been carried out a posteriori. The width of the joint (at the surface) and its cross section for each metallic component have been determined (for the weld seams " 2 " to "6"), Figure 7. The dilution percentage of E24 steel in the weld is obtained from the ratio of E24 cross-section to the whole cross-section of the weld seam, Figure 6 . The calculation can be done for the mixing of two different metal plates or, as we will see further, for the deposition of Inconel wire on a plate during welding. The dilution can be expressed as:

$$
\text { dilution }=\frac{\text { area } 1}{\text { area } 1+\text { area } 2} .
$$

We can observe on these two graphs that the cross section variations of the weld joint seem to be linked to the

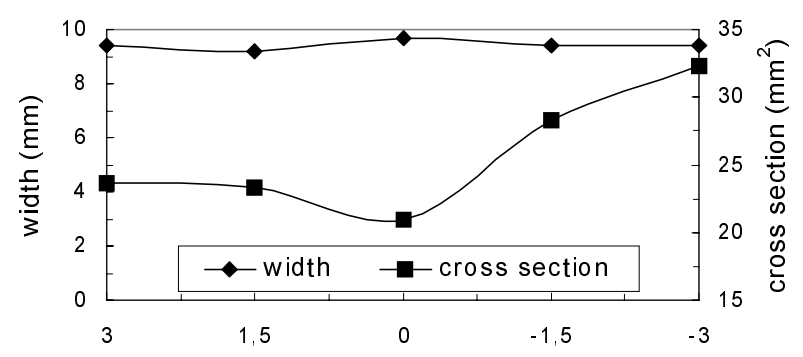

electrode position to the center of the plates $(\mathrm{mm})$

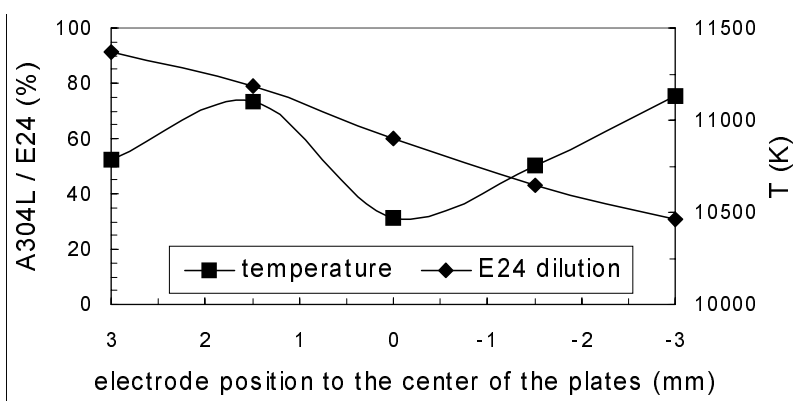

Fig. 7. Width, cross-section, dilution and temperature.

evolution of the plume temperature. In this experiment the current intensity remains constant and the arc radius does not vary which explains the constant width at the metal surface [21]. The width of the seam remains almost unchanged (around $9.5 \mathrm{~mm}$ ). When the temperature rises for a constant width of the weld joint at the metal surface, the cross-section of the seam should be greater. For example, if we consider the weld seam " 4 " the minimum cross-section $\left(20.93 \mathrm{~mm}^{2}\right)$ is reached for the lower temperature $(10470 \mathrm{~K})$. However, it is not easy to link accurately the calculated temperatures to the cross-section. The weld seam joint has an approximate length of $6 \mathrm{~cm}$ which corresponds to more then $70 \mathrm{~s}$ of welding, and the temperature is obtained for a spectrum acquisition time of $1 \mathrm{~s}$. This is why we considered another technique to monitor the weld seam dilution.

On the other hand, this graph shows clearly that there is more E24 steel in the seam than A304L stainless steel. This phenomenon is also observed in the case of E24 steel and Inconel welding. It might be due to the fact that E24 steel thermal conductivity is higher than those of Inconel and A304L.

We verify dilution by studying the emission line variations for the different elements present in the two plates. For example, in a given spectral range, we can follow lines of argon, iron and nickel. Their relative intensity variations might inform us about the dilution in the weld. Figure 8 exhibits 130 spectra successively recorded in the same wavelength range during the realisation of the weld seam " 8 " for the E24 and Inconel plates. A small part of the studied wavelength range is magnified to follow the emission line variations.

At the beginning of the experiment we have E24 steel (spectrum No. 1), at the end we only have Inconel (spectrum No. 130). Some emission lines have characteristic 


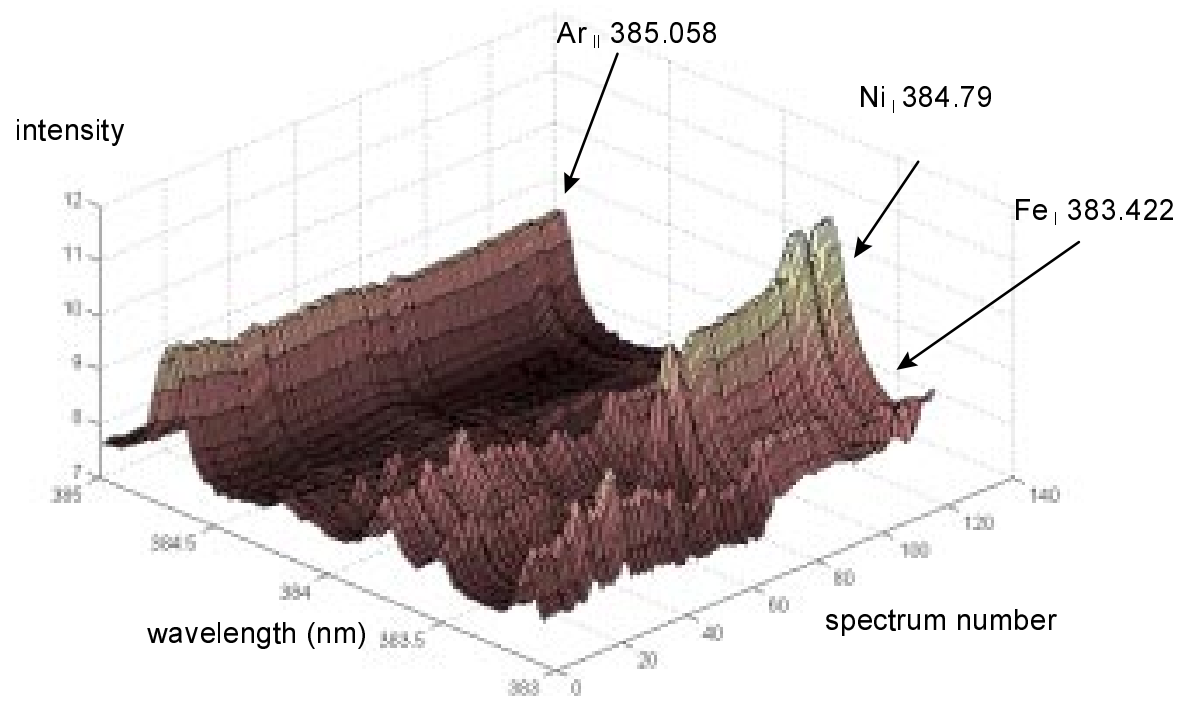

Fig. 8. Dilution spectra of E24 steel into Inconel with argon shielding.

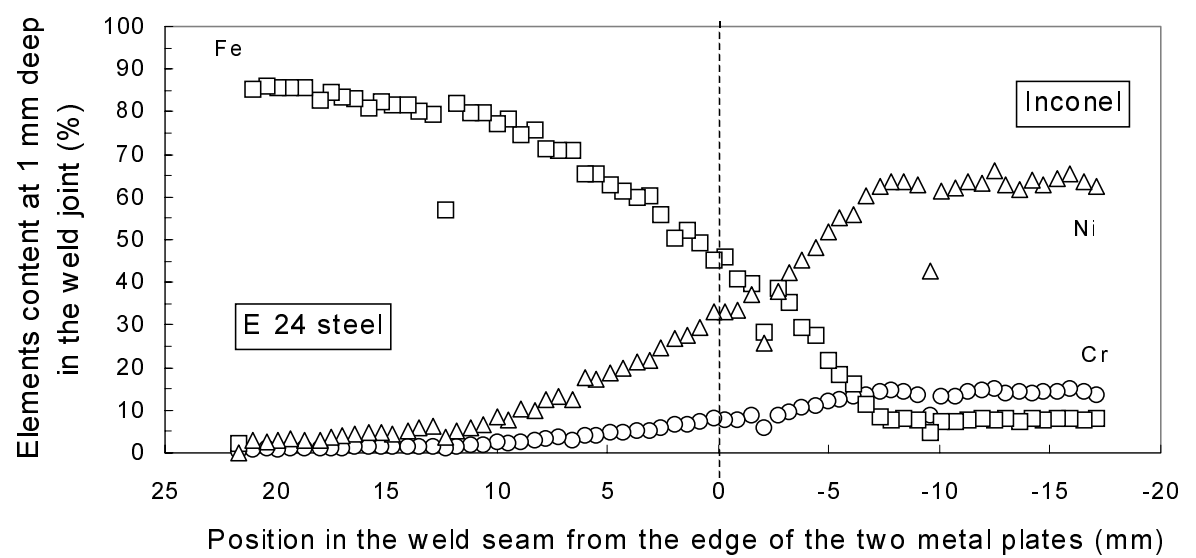

Fig. 9. Metallographic analysis at one millimetre deep in the weld seam obtained during the welding of an E24 steel to an Inconel plate.

shapes. The $\operatorname{Ar}_{I I} 385.058$ line (singly ionised argon) remains constant during all the process, there is only a small signal increase in the first spectra due to the instability of the plasma plume. The iron line $\mathrm{Fe}_{\mathrm{I}} 383.422$ has another profile which has been observed for all of the iron lines. A remarkable fact is the emergence of the $\mathrm{Ni}_{\mathrm{I}} 383.79$ line which is very strong in the last spectra. This is due to the fact that the Inconel is a nickel based alloy. A metallurgical analysis of the main chemical elements present in the weld seam "8" was performed. Figure 9 presents the contents of iron, chromium and nickel at one millimetre deep inside the weld joint. The relative content of each component is plotted versus the distance to the contact surface of the two plates. We can see that the element content does not change quickly as in the case of spectroscopic measurements. This can be explained by the fact that molten pool hydrodynamic in front of and behind the induced arc plasma mixes the two metals. This experiment shows that the monitoring of the dilution of two different metals during welding can be carried out. We will deal about this now.

\subsection{Welding with metallic wire}

Our last spectroscopic measurements concern the welding of stainless and E24 steel with an Inconel 82 wire. A GTAW machine is used with a special tool providing the wire (diameter $0.9 \mathrm{~mm}$ ) at various speeds, from 0 to $2.54 \mathrm{~m} / \mathrm{min}$. The choice of E24 rather than stainless steel or Inconel can be justified by the stronger contrast existing between the atomic spectra of the wire and the plate components. In the case of industrial applications Inconel wires are often used with stainless steel.

First we observed the influence of the wire speed deposition which is directly related to the amount of Inconel melt in the weld seam. The wavelength range recorded to determine the plasma temperature (Sect. 3.2) is still valid for following spectral intensities of nickel and chromium emission lines which are also present in this area.

The calculated temperature (Fig. 10), between 11300 and $12100 \mathrm{~K}$, grows with the wire speed up to $2 \mathrm{~m}$ per minute. However, at $2.54 \mathrm{~m}$ per minute the value of $T$ is very low and must be erroneous (strong instabilities 


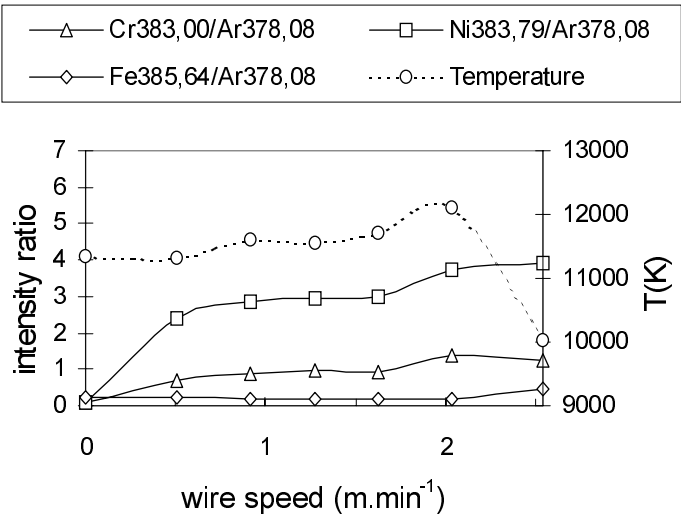

Fig. 10. Line intensity ratios and temperature versus wire speed.

of the plasma plume). Furthermore the plasma plume temperature is greater in the case of iron welding with alloy elements (nickel and chromium) as we showed before. In order to control the rate of wire deposition in the weld seam it is of interest to follow the intensity of characteristic emission lines. We have noted that gas emission lines (argon) remain constant. It is clearer to follow the ratio of a metallic line in relation to an argon line. We chose the $378.084 \mathrm{~nm}$ singly ionised argon line as reference.

The strongest alloy emission line observed is the nickel one at $383.79 \mathrm{~nm}$. We also observed the chromium line at $383.003 \mathrm{~nm}$ and the iron line at $385.637 \mathrm{~nm}$. We can see that the nickel and chromium line intensities increase with the speed of the wire, in fact with the amount of Inconel in the weld seam. The iron line intensity remains almost constant despite the increase of wire speed, the iron being present only in the metal piece (a few percent in the wire).

We have correlated the spectroscopic measurements with microphotographs of the fused area of the seams. The metallographic analysis of the welds allows us to calculate the amount of iron, nickel and chromium in the fused zone (Fig. 11). Comparison of spectroscopic and metallographic results shows that the trends are similar, especially at higher deposition speeds. We remark that the elemental composition obtained from the metallurgical analysis was measured one time in the cross section of each weld seam. It is not obvious to relate spectroscopic measurements (average value over 1 second acquisition time) for low speeds of wire deposition. We think that it would be of interest to replace the PDA detector by a more sensitive acquisition device such as a photo-multiplier, which has a larger dynamic response, focused on an characteristic emission line. This kind of apparatus could be useful as a remote sensor to monitor the dilution in the weld seam, the fulfilment of a good dilution (not only a "pasting") between the two metals being a major criterion of quality.

\section{Conclusion}

This study presents some aspects of spectroscopic measurements related to the plasma induced during arc weld-

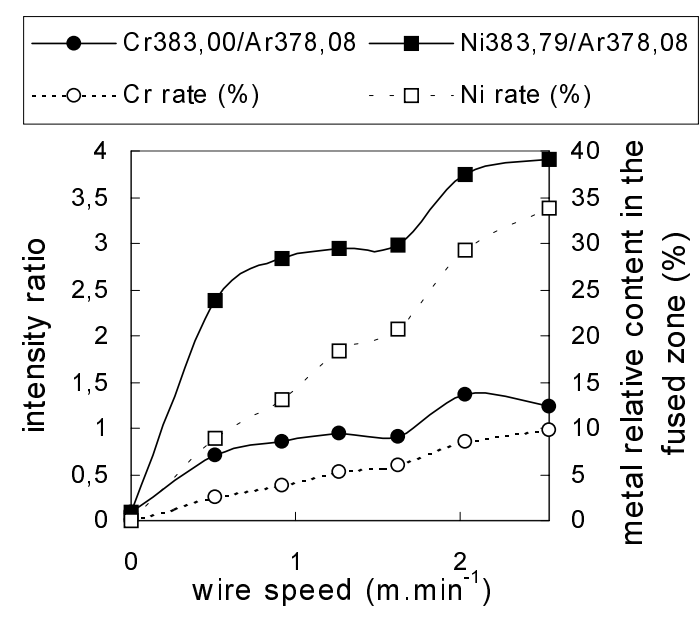

Fig. 11. Dilution monitoring, spectroscopic and metallographic results.

ing. We first presented the experimental set-up and some results concerning metallic and gas plasmas. Then we used the Boltzmann statistics to compute the electron temperature of the medium for several process parameters and different metals.

We have shown that the plasma temperature is very sensitive to the material components, being higher in the case of alloys which contain chromium. On the other hand, the arc-induced plasma temperature does not vary significantly with the supplied power (in the range 2100$2800 \mathrm{~W}$ ).

The last experiments have dealt with the dilution between two distinct metals. We have observed that the weld cross-section is linked to the plasma temperature. We remark that the dilution inside the weld seam should be checked following the relative intensity of different metal emission lines, such as iron and nickel or iron and chromium. Further experiments will be necessary to confirm this observation. Finally we dealt with the evolution of the dilution of a Inconel wire in the weld joint. We showed that a suitable detection device could be used to check the dilution of a wire. This is very important for the welding of thick metal pieces, such as those used in nuclear constructions where no defects in the joining can be accepted.

The authors wish to thank the FRAMATOME team of St. Marcel for helping them use the GTA and GMA devices, and for the preparation of the experiments.

\section{References}

1. J.J. Lowke, R. Morrow, J. Haidar, A.B. Murphy, IEEE Trans. Plasma Sci. 25, 925 (1997).

2. J. Haidar, J.J. Lowke, J. Phys. D: Appl. Phys. 29, 2951 (1996).

3. H.G. Fan, S.J. Na, Y.W. Shi, J. Phys. D: Appl. Phys. 30, 94 (1997). 
4. P.G. Jönsson, R.C. Westhoff, J. Szekely, J. Appl. Phys. 74, 5997 (1993).

5. D. Saini, S. Floyd, Welding J. 77, 172 (1998).

6. T.A. Siewert, R.B. Madigan, T.P. Quinn, Adv. Mat. Proc. 151, 23 (1997).

7. C.W. Lee, S.J. Na, Welding J. 75, 379 (1996).

8. K.M. Yun, S.J. Na, Welding J. 70, 43 (1991).

9. M. Onsøien, R. Peters, D.L. Olson, S. Liu, Welding J. 74, 10 (1995).

10. Q. Pang, T. Pang, J.C. Mc Clure, A.C. Nunes, J. Eng. Ind. 115, 145 (1993).

11. M.M. Collur, T. Debroy, Metall. Trans. B 20, 277 (1989).

12. D. Lacroix, G. Jeandel, C. Boudot, J. Appl. Phys. 81, 6599 (1997).

13. A. Block-Bolten, T.W. Eagar, Trends in Welding Research in the United States, edited by S.A. David (ASM Metals Park, Ohio, 1982), pp. 53-73.

14. R.W. Richardson, Characterization of welding arc spectral light emission, IIW Doc. XII-1469-96, 49th Annual assembly of the International Institute of Welding, Budapest, 31 Aug. -7 Sept. 1996.

15. H. Griem, Plasma Spectroscopy (Mc Graw-Hill, New York, 1964).

16. G.V. Marr, Plasma Spectroscopy (Elsevier, London, 1968).

17. J.R. Fuhr, G.A. Martin, W.L. Wiese, J. Phys. Chem. Ref. Data 17, 305 (1988).

18. D.E. Blackwell, P.A. Ibbetson, A.D. Petford, M.J. Shallis, MNRAS 186, 633 (1979).

19. D.M. Evans, D. Huang, J.C. McClure, A.C. Nunes, Welding J. 77, 53s (1998).

20. P. Vervisch, M. Berrichi, J.F. Lhuissier, B. Cheron, M. Terrier, Étude physique et mécanique d'un arc TIG par diagnostiques optiques, Soudage et Techniques Connexes, pp. 5-11, January-February 1985.

21. J.F. Key, J.W. Chan, M.E. Mc Ilwain, Welding J. 62, 179s (1983). 